\title{
1.1 Head-on beam-beam compensation in RHIC
}

\author{
W. Fischer, Y. Luo, A. Pikin, E. Beebe, D. Bruno, D. Gassner, X. Gu, R.C. Gupta, \\ J. Hock. A. Jain, R. Lambiase, M. Mapes, W. Meng, C. Montag, B. Oerter, M. \\ Okamura, D. Raparia, Y. Tan, R. Than, J. Tuozzolo, and W. Zhang \\ Mail to: wfischer@bnl.gov \\ Brookhaven National Laboratory, Upton, NY 11973, USA
}

\subsubsection{Introduction}

Head-on beam-beam compensation with electron lenses had been proposed for the SSC, LHC, and the Tevatron [1,2]. Two electron lenses are installed in the Tevatron [2-4], where they are routinely used as gap cleaner and have been tested in many other configurations. In RHIC there are 2 head-on beam-beam interactions at IP6 and IP8, and 4 long-range beam-beam interactions with large separation $(10 \mathrm{~mm})$ at the other IPs.

We consider the partial indirect compensation of the head-on beam-beam effect with one electron lens in each ring. Together with intensity and emittance upgrades $[5,6]$ our goal is to approximately double the luminosity over what can be achieved without these upgrades. A RHIC electron lens consists of: a DC electron gun, an electron beam transport to the main solenoid, the superconducting main solenoid in which the interaction with the hadron beam occurs, an electron beam transport to the collector, and an electron collector. The 2 electron lenses are located in IR10 between the DX beam separation dipoles. The proton beams pass through the main solenoids of both electron lenses, and interact head-on with one of them. The following is a slightly modified version of Ref. [7]. The table shows the main parameters of the proton beam and the electron lenses. References [8-11] present simulations for and discuss beam dynamics problems.

\subsubsection{Design considerations}

We aim for a technically feasible and economically viable implementation that comes as close as possible to the ideal compensation scheme: A phase advance of $\pi$ between $\mathrm{p}-\mathrm{p}$ and $\mathrm{p}$-e interactions for all betatron amplitudes and the same amplitude dependent beam-beam kick from the $\mathrm{p}-\mathrm{p}$ and $\mathrm{p}$-e interaction. In addition, the ease of commissioning and operation is a design consideration. 


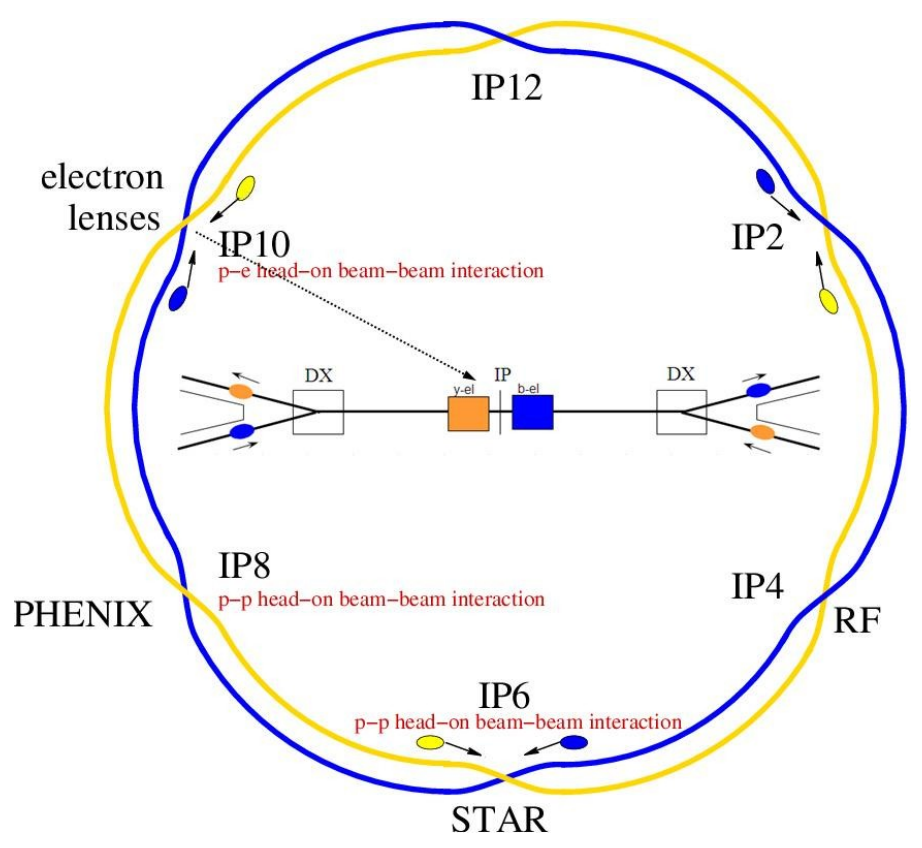

Figure 1: Layout for head-on beam-beam compensation in RHIC. Head-on beambeam interactions are in IP6 and IP8, compensation near IP10.

Nonlinearities between the p-p and p-e interactions are minimized when these are as close as possible (see figure). Phase advances of $\pi$ are realized through lattice design and a phase shifter. To obtain the same amplitude dependent forces, the location is limited to the space between the DX magnets, where the dispersion is also small: Since the proton beams are round in the beam-beam interactions, we also require $\beta_{x}=\beta_{y}$ at the electron lens and matched transverse proton and electron beam profiles.

The tolerances for the main solenoid field straightness and the tolerances for the relative beam alignment are easier to meet with a larger proton beam. The $\beta$-function at IP10 can be as large as $10 \mathrm{~m}$.

The electron beam size in the main solenoid $\sigma_{\mathrm{e}}$ is given by its size at the cathode $\sigma_{\mathrm{ec}}$, the solenoid fields at the cathode $B_{\mathrm{sc}}$, and in the main solenoid $B_{\mathrm{sm}}: \sigma_{\mathrm{e}}=$ $\sigma_{\mathrm{ec}} \mathrm{Q}\left(B_{\mathrm{sc}} / B_{\mathrm{sm}}\right)^{1 / 2} . B_{\mathrm{sm}}$ is limited to about $6 \mathrm{~T}$, and a strong field makes a correction of the field straightness difficult. $B_{\mathrm{sc}}$ has to be large enough to suppress space charge effects. With these limits, and a given beam size $\sigma_{\mathrm{e}}$ the electron beam size and current density at the cathode follow, and must be technically feasible. We use a DC electron beam to minimize the noise in the electron beam.

With both electron lenses in IR10, in a section common to both beams (see figure), the effect of the main solenoids on coupling and spin orientation are locally compensated.

The instrumentation must allow monitoring of the electron beam current and shape, and the relative position and angle of the electron and proton beams. In a diagnostics mode the electron beam current is modulated. 
Table 1: Reference case for RHIC beam-beam and beam-lens interactions.

\begin{tabular}{|c|c|c|}
\hline Parameter & Unit & Value \\
\hline \multicolumn{3}{|l|}{ Proton beam parameters } \\
\hline Total energy $E_{\mathrm{p}}$ & $\mathrm{GeV}$ & 250 \\
\hline Relativistic factor $\beta_{\mathrm{p}}$ & $\cdots$ & 0.999993 \\
\hline Relativistic factor $\gamma_{p}$ & $\ldots$ & 266.4 \\
\hline Bunch intensity $N_{\mathrm{p}}$ & $10^{11}$ & 2.0 \\
\hline$\beta_{x, y}^{*}$ at IP6, IP8 (p-p) & $\mathrm{M}$ & 0.5 \\
\hline$\beta_{\mathrm{x}, \mathrm{y}}^{*}$ at IP10 (p-e) & $\mathrm{M}$ & 10.0 \\
\hline Lattice tunes $\left(Q_{\mathrm{x}}, Q_{\mathrm{y}}\right)$ & $\cdots$ & $(.695, .685)$ \\
\hline Rms emittance $\varepsilon_{\mathrm{n}}$, initial & $\mathrm{mm} \mathrm{mrad}$ & 2.5 \\
\hline Rms beam size at IP6, IP8, $\sigma_{p}^{*}$ & $\mu \mathrm{m}$ & 70 \\
\hline Rms beam size at IP10, $\sigma_{p}^{*}$ & $\mu \mathrm{m}$ & 310 \\
\hline Rms bunch length $\sigma_{\mathrm{s}}$ & M & 0.25 \\
\hline Rms momentum spread $\delta p / p$ & $10^{-3}$ & 0.30 \\
\hline Hourglass factor $F$, initial & $\ldots$ & 0.88 \\
\hline Beam-beam parameter $\xi / I P$ & $\ldots$ & 0.010 \\
\hline Number of beam-beam IPs & $\ldots$ & $2+1$ \\
\hline \multicolumn{3}{|l|}{ Electron lens parameters } \\
\hline Distance of center from IP & M & 2.0 \\
\hline Effective length $L_{\mathrm{e}}$ & $\mathrm{M}$ & 2.1 \\
\hline Kinetic energy $E_{\mathrm{e}}$ & $\mathrm{kV}$ & 6.4 \\
\hline Relativistic factor $\beta_{\mathrm{e}}$ & $\ldots$ & 0.16 \\
\hline Relativistic factor $\gamma_{\mathrm{e}}$ & $\cdots$ & 1.013 \\
\hline Electron line density $n_{\mathrm{e}}$ & $10^{11} \mathrm{~m}^{-1}$ & 0.82 \\
\hline Electrons in lens $N_{\mathrm{e} 1}$ & $10^{11}$ & 1.7 \\
\hline Electrons encountered $N_{\mathrm{e} 2}$ & $10^{11}$ & 2.0 \\
\hline Current $I_{\mathrm{e}}$ & A & 0.62 \\
\hline
\end{tabular}

Electron gun. The gun has to provide a beam with a transverse profile that is close to Gaussian. Considering the magnetic compression of the electron beam into the main solenoid center with maximum magnetic field of $6 \mathrm{~T}$ the a cathode diameter of $7.0 \mathrm{~mm}$ gives a Gaussian profile with $2.8 \mathrm{rms}$ beam sizes. The perveance of the gun is $P_{\text {gun }}=0.86 \square 10^{-6} \mathrm{AV}^{-1.5}$. The current density of the electron beam on its radial periphery can be changed with the control electrode voltage while the general shape of the beam profile remains Gaussian. The cathode should be capable of providing an emission current density of $21 \mathrm{Acm}^{-2}$ for an electron beam current of $1 \mathrm{~A}$ continuously and in a regime of short pulses with a reasonable life time of several thousand hours. Cathodes made of IrCe satisfy these requirements. 


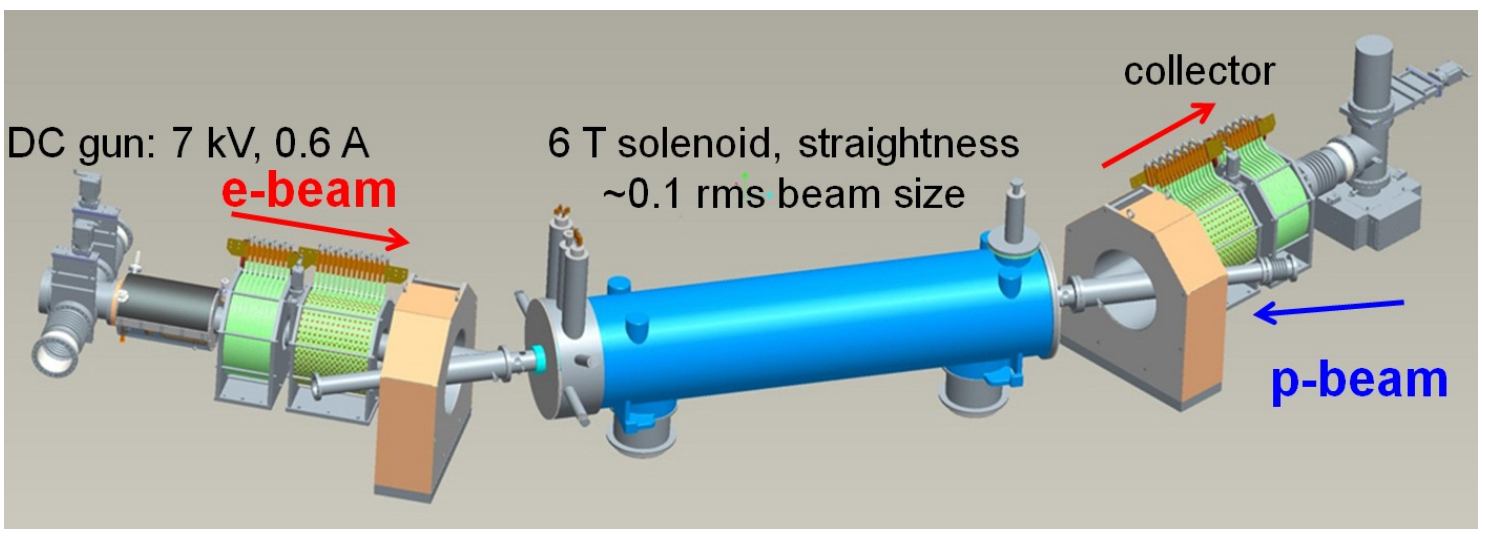

Figure 2: Electron lens layout with gun, electron beam transport, superconducting main solenoid for interaction with the proton beam, and electron collector.

Transport solenoids and steering magnets. The electron beam is transported from the gun to the main solenoid through two solenoids (see figure) which provide focusing. Within the second solenoid horizontal and vertical steering magnets are incorporated that can move the beam by $5 \mathrm{~mm}$ in either plane. The power consumption of both electron lenses should be limited to a total of $500 \mathrm{~kW}$ in order to avoid upgrades to the electrical and water cooling infrastructure in IR10.

Superconducting main solenoid. The superconducting main solenoid provides the interaction region between the electron and the proton beam. The length and the electron beam current define the interaction strength. The field strength $(6 \mathrm{~T})$, together with the field solenoid field strength at the gun define the transverse beam size, that must match the proton beam size. The field lines in the solenoid should not deviate from a straight line by more than $10 \%$ of an rms beam size (see table). This requires 5 dipole correctors in each plane that will increase the straightness of the cold solenoid by a factor 4-5. A horizontal and vertical angle corrector for the electron beam will also be implemented. All dipole corrector are superconducting magnets, wound on top of the main solenoid, and housed in the same cryostat.

Electron collector. The electron collector has to dissipate the power of the electron beam coming from the interaction region. The nominal current of the electron beam is $0.6 \mathrm{~A}$ and the maximum is close to $2 \mathrm{~A}$. The design is dictated primarily by the UHV requirements of RHIC. It allows the separation of the heavily bombarded area from the rest of the electron lens by using a small diaphragm. A magnetic shield leads to fast diverting electrons inside the collector. The reflector has a potential lower than the cathode and pushes electrons outwards to the water-cooled cylindrical surface. Under a load twice as high as expected from a 2 A electron beam the maximum temperature on inner surface of the shell is $102 \circ \mathrm{C}$. This temperature is acceptable for the material (copper) and for UHV conditions in RHIC. 20 tubes with an ID of $8 \mathrm{~mm}$ are brazed to the outside of the cylindrical shell and are connected in parallel for water flow.

Diagnostics. In commissioning mode an electron current modulator is used so that the beam is on only during the abort gap or a few selected RHIC bunches. In compensation mode the electron beam is DC. The goal is to align a small proton beam inside an only slightly larger electron beam within about $10 \%$ of an rms beam size. BPMs are used to bring both beams close together, while bremsstrahlung detectors [11] and proton beam diagnostics are used to maximize the overlap of the beams. Due to the large difference 
in electron $(1 \mu \mathrm{m})$ and proton beam bunch lengths ( $5 \mathrm{~ns})$, separate electron and proton beam position monitors are needed. The electron beam needs to be monitored for shape, current, and loss. Multipurpose isolated electrodes will be mounted throughout electron beam transport. The electrodes detect lost beam, and clear ions.

Power supplies. Most of the supplies are referenced to ground; elements in the gun and collector are referenced to the cathode. The gun supplies include the cathode bias supply, the cathode heater, the beam forming supply, and two anode supplies (DC and pulsed). A cathode, focusing, and bending solenoid are powered individually. There are also four sets of dipole windings for each lens. The superconducting solenoid has a power supply that allows slow ramping only. Within the superconducting solenoid, eight drift tubes and twenty trim magnets are powered individually. The collector power supply is rated with $10 \mathrm{kV}$ at $2 \mathrm{~A}$, and will limit the energy deposited in the device should an arc occur. An ion extractor is powered with respect to the cathode potential. A suppressor element is powered with respect to the collector.

Vacuum system. The gun and collector vacuum will be UHV compatible, with a design pressure of $10^{-10}$ Torr, and interface to the RHIC warm bore with a nominal pressure of $10^{-11}$ Torr. For this reason all of the components shall be bakable to $250 \circ \mathrm{C}$. All-metal gate valves separate the gun and collector vacuum. The gun and collector chambers will have a confined gas load by using a conductance limiting aperture, and enough installed pumping speed. All vacuum chambers interfacing with the RHIC warm bore will be made from stainless steel and have NEG coating.

\subsubsection{Summary}

Partial head-on beam-beam compensation is planned in RHIC with electron lenses. The main solenoid, electron gun and collector, the electron beam transport, and instrumentation are designed and under construction. We expect the completion of the hardware installation at the end of 2012 expect a luminosity gain of up to a factor of two together with a polarized proton source upgrade $[5,6]$ that can deliver higher bunch intensities.

\subsubsection{Acknowledgments}

We are thankful for discussion and support to many, in particular N. Milas, J. Alessi, M. Anerella, A. Fedotov, G. Ganetis, E. Haug, W. Herr, V. Kamerdzhiev, J.-P. Koutchouk, G. Kuznezov, W. Nakel, K. Ohmi, T. Roser, T. Sen, L. Snydstrup, V. Shiltsev, G. Sterbini, P. Thieberger, A. Valishev, and F. Zimmermann. This article has been authored by employees of Brookhaven Science Associates, LLC under Contract No. DE-AC0298CH10886 with the U.S. Department of Energy. The United States Government retains a non-exclusive, paid-up irrevocable, world-wide license to publish or reproduce the published form of this article, or allow others to do so, for the United States Government purposes.

\subsubsection{References}

1. E. Tsyganov, R. Meinke, W. Nexsen, A. Zinchenko, "Compensation of the beam-beam effect in proton colliders", SSCL-PREPRINT-519 (1993).

2. V. Shiltsev, V. Danilov, D. Finley, and A. Sery, "Considerations on compensation of beam-beam effects in the Tevatron with electron beams", Phys. Rev. ST - Accel. Beams 2, 071001 (1999).

3. V. Shiltsev, Y. Alexahin, K. Bishofberger, V. Kamerdzhiev, G. Kuznetsov, and 
X.-L. Zhang, "Experimental demonstration of colliding-beam-lifetime improvement by electron lenses", Phys. Rev. Lett. 99, 244801 (2007).

4. V. Shiltsev et al., "Tevatron electron lenses: Design and operation", Phys. Rev. ST Accel. Beams 11, 103501 (2008).

5. D. Raparia et al., "Results of LEBT/MEBT reconfiguration at BNL $200 \mathrm{MeV}$ LINAC", PAC'09, submitted to Phys. Rev. ST - Accel. Beams (2009).

6. A. Zelenski, "Review of polarized ion sources", Rev. Sci. Instrum. 81 02B308 (2010).

7. W. Fischer et al., "Status of the RHIC head-on beam-beam compensation project", Proc. IPAC10.

8. W. Fischer, Y. Luo, C. Montag, "Bunch length effects in the beam-beam compensation with an electron"', Proc. IPAC2010

9. C. Montag et al., "Ion bunch length effects on the beam-beam interaction in a high luminosity ring-ring electron-ion collider with head-on beam-beam compensation", Proc. IPAC2010.

10. Y. Luo and W. Fischer, "6-D weak-strong simulation of head-on beam-beam compensation in RHIC", Proc. IPAC2010.

11. Y. Luo, "SimTrack: a simple $\mathrm{C}++$ library for particle tracking", Proc. IPAC2010.

12. C. Montag et al., "Optimizing the beam-beam alignment in an electron lens using bremsstrahlung”, Proc. IPAC2010. 